\title{
Erfassung der Lebenswelt mittels Mobility Mapping: Einsatz einer visuellen Methode als jahrgangsübergreifende Lehr- Lern-Sequenz
}

\author{
"stefan.kordel@fau.de, Institut für Geographie, Universität Erlangen-Nürnberg ( $\square$ korresp. Autor) \\ ** tobias.weidinger@fau.de, Institut für Geographie, Universität Erlangen-Nürnberg \\ ${ }^{* * *}$ manuela.kocher@jisbg.de, Johann-Sebastian-Bach-Gymnasium Windsbach
}

eingereicht am: 30.04.2021, akzeptiert am: 08.09.2021

\begin{abstract}
Mobility Mapping ist eine Forschungsmethode, die die räumliche Dimension der Lebenswelt von Individuen oder Gruppen mithilfe einer von Teilnehmenden selbst erstellten Karte erforscht, um Einblick in deren bedeutsame Orte sowie Mobilitätspraktiken zu erhalten. Aufgrund der vielfältigen Lernziele für und die Orientierung auf Teilnehmende hat die visuelle Methode auch ein großes Potenzial für den Geographieunterricht. Der Transfer von der Fachwissenschaft in die schulische Praxis erfolgt im Beitrag im Rahmen einer Lehr-Lern-Sequenz mit Projektcharakter, bei der Schüler*innen der Oberstufe Mobility Maps vorbereiten und mit Schüler*innen der Unterstufe in Kleingruppen durchführen und auswerten.
\end{abstract}

Keywords: Raumkonstruktion, Karten, Lebenswelt, Alltagsmobilität, Reflexivität, Orientierung

\section{Capturing the lifeworld by means of mobility mapping: Using a visual method as part of an inter- year teaching-learning sequence}

Mobility Mapping is a research method that explores the spatial dimension of the lifeworld of individuals or groups by means of a map created by participants themselves in order to gain insight into their important places as well as mobility practices. Due to the diverse learning objectives for and orientation towards participants, the visual method also has great potential for geography teaching. In the article, the transfer from science to school practice is exemplified by a teaching-learning sequence with project character, in which small groups of students from upper secondary classes prepare Mobility Maps, carry them out with students of lower secondary classes and jointly evaluate them.

Keywords: construction of space/place, maps, lifeworld, everyday mobility, reflexivity, orientation

\section{$1 \quad$ Einführung}

Die raumbezogene Forschungsmethode Mobility Mapping bietet einen Zugang zu Lebenswelten von Individuen. Mittels Kartierung der eigenen sozialräumlichen Alltagswelt wird ein visuelles Produkt erstellt, das anschließend gemeinsam mit den Forschenden ausgewertet werden kann. Ein Vorteil dieser Methode ist vor allem, dass sie eine Ausdrucksmöglichkeit über die verbale hinaus bietet. Außerdem nehmen Forschungsteilnehmende, dem partizipativen Paradigma qualitativer Forschung entsprechend, an der Erhebung und Analyse der Daten teil und Machtasymmetrien zwischen Forschenden und Teilnehmenden lassen sich abbauen.
Eine Anwendung des Mobility Mapping im schulischen Unterricht wird von den Autor*innen als lernfördernd erachtet, da damit eine Schüler*innenorientierung einhergeht und sich vielfältige Lernziele erfüllen lassen. So können Schüler*innen geographische Arbeitsweisen und Fachmethoden einüben (instrumentales/instrumentelles Lernziel), ihre Erfahrungen aus der eigenen Lebenswelt einbringen (affektives Lernziel) und über die „Gemachtheit“ der Welt und ihre sozialräumliche Interaktionen reflektieren und ihre räumlichen Kompetenzen erweitern (kognitives Lernziel) (Rinschede \& Siegmund 2020: $135 \mathrm{f}$.).

Im vorliegenden Beitrag wird zunächst das visuell- 
partizipative Forschungsinstrument Mobility Mapping im Hinblick auf Potenziale und Herausforderungen vorgestellt. Dabei wird auf eine empirische Fallstudie Bezug genommen, die mit neuzugewanderten Geflüchteten durchgeführt wurde. Der Transfer in den Unterrichtskontext wird anschließend am Beispiel einer didaktischen Umsetzung in einer LehrLern-Sequenz dargestellt. Dabei stehen Lebenswelten von Kindern und Jugendlichen und die Konstruktion von städtischen und ländlichen Räumen im Mittelpunkt. Zielgruppen sind Schüler*innen der Oberstufe, die Mobility Maps vorbereiten und mit Schüler*innen der Unterstufe in Kleingruppen durchführen und auswerten.

\section{Mobility Mapping als humangeographi- sche Forschungsmethode}

Seit den 1980er-Jahren werden in der sozialwissenschaftlichen Forschung im Allgemeinen und der humangeographischen Forschung im Speziellen visuelle Methoden eingesetzt (vgl. Collier \& Collier 1986; Rose 2007; Schlottmann \& Miggelbrink 2009; Spencer 2011). Dabei wirkten zuletzt auch Teilnehmende selbst aktiv an der Produktion von visuellen Daten mit, wie dies etwa bei der Fotoelizitation der Fall ist (vgl. Kordel 2015). Im Kontext der development studies (Entwicklungsforschung) entstanden (Kumar 2002), zielt Mobility Mapping als raumbezogenes Erhebungsinstrument darauf ab, die räumliche Dimension von Lebenswelten von Individuen oder Gruppen mithilfe einer Karte, die durch Teilnehmende selbst erstellt wird (Mobility Map), zu erforschen. Das Konzept Lebenswelt bezieht dabei neben aktuellem Alltagshandeln auch Erfahrungen der Vergangenheit und Erwartungen an die Zukunft ein (Honer 2011). Subjektive und gesellschaftliche Wissensvorräte sowie deren Wechselwirkungen helfen Individuen, sich in der Lebenswelt zu orientieren (Schütz \& Luckmann 1979). Bei der Erstellung der Karte schreiben die Teilnehmenden Orten eine Bedeutung zu und reflektieren darüber, wie diese erreicht werden können. Dabei sind subjektiv wahrgenommene Distanzen und Erreichbarkeiten, aber auch Fragen des Nicht-Zugangs zu diesen Orten als Formen der Exklusion aufgrund fehlender zeitlicher oder finanzieller Ressourcen, rechtlicher Gründe oder gesundheitlicher Einschränkungen, von Relevanz (Weidinger et al. 2019).

Mobility Mapping nimmt zum einen Bezug auf Mental Maps, die in der Geographie bereits seit den 1960er-Jahren eingesetzt werden, wird aber um die Wahrnehmung von Wegen erweitert (vgl. Route Mapping, Morojele \& Muthukrishna 2012). Zum anderen werden die erstellten Karten mit Narrationen verknüpft (vgl. Narrative Landkarten, Lutz et al. 2003). Während des Gesprächs wechseln sich Sequenzen des Zeichnens und narrative Sequenzen ab. Durch die Reflexion der Teilnehmenden mit der forschenden Person über das Gesagte bzw. Gezeichnete fällt beim $\mathrm{Mo}$ bility Mapping die Phase der Durchführung mit der der Auswertung zusammen und stärkt deshalb den partizipativen Charakter der Methode. Zudem können intertextuelle Bezüge zwischen dem Kartenprodukt (Text 1) und dem gesprochenen Wort (Text 2) herausgearbeitet werden.

In einem von Weidinger, Kordel und Kieslinger (2019) durchgeführten Mobility Mapping mit Geflüchteten wurden die Teilnehmenden eingeladen, Orte und Plätze, die sie aufsuchen und die im Lebensalltag wichtig sind, auf Handzettel zu schreiben und sie entsprechend ihrer Distanz zur Wohnung auf einem Plakat zu verteilen. Teilnehmende wurden insbesondere dazu ermutigt, eigene Zeichnungen zu erstellen und Beschriftungen vorzunehmen (vgl. auch Erfahrungen bei Wilde 2014). Sie wurden beim Schreiben und Zeichnen nicht unterbrochen, um ihre aktive Mitgestaltung zu fördern und ihr individuelles Arbeitstempo zu berücksichtigen. Erst anschließend wurden sie gebeten, Orte zu ergänzen, die sie in ihren Erzählungen bereits erwähnt, aber noch nicht aufgezeichnet hatten. Als Erinnerungsstütze dienten kleine Karten, die mit Piktogrammen zu verschiedenen Bereichen des Alltagslebens (z. B. Einkaufen, Freizeit, Bildung und Arbeit) versehen waren. In der darauffolgenden Erzählsequenz stand die Bedeutung der gezeichneten Orte im Vordergrund, wie z. B. die Gründe, diese aufzusuchen, vor Ort durchgeführte Aktivitäten, die Dauer und Häufigkeit der Aufenthalte, begleitende Personen und die benutzten Verkehrsmittel, um diese Orte zu erreichen. Ein inhaltlicher Schwerpunkt lag somit auf den eigenen Erfahrungen, spezifischen Ereignissen und Situationen. Zum Schluss des Gesprächs wurden in den Mobility Maps auch diejenigen Orte aufgenommen, die die Teilnehmenden aus verschiedenen Gründen nicht aufsuchen wollten, konnten oder durften (vgl. Täubig 2009; Sampson \& Gifford 2010). Abschließend wurde ein Foto des fertigen Zeichenprodukts erstellt und die Karte den Teilnehmenden übergeben.

Für eine anschließende, forscher*innengeleitete Analyse von Mobility Maps schlagen Lutz, Behnken und Zinnecker (2003) grundsätzlich drei Auswertungsmodi vor. Der erste Modus fokussiert eine prozessorientierte Herangehensweise. Während des Zeichnens ,laufen' die Teilnehmenden Wege und Orte in Gedanken ab. Deshalb erlaubt die Analyse der Chronologie des Zeichenprozesses Rückschlüsse auf zeitliche Abfolgen von Alltagspraktiken der Befragten, 
z. B. den Weg von der Unterkunft zum Sprachkurs am Vormittag und den Rückweg nach dem Einkauf am Nachmittag. Hinweise auf die Relevanz von bestimmten Orten für das Individuum können erlangt werden, indem zwischen Orten unterschieden wird, die von Teilnehmenden ad hoc und auf Nachfrage mithilfe der Erinnerungsstützen gezeichnet wurden. Zusätzlich kann durch die Audioaufnahme die zeitliche Dauer der Erzählung über einen Ort Berücksichtigung finden.

Videoaufnahmen des Zeichenprozesses erleichtern die prozessorientierte Analyse, sind jedoch mit spezifischen Herausforderungen verbunden, z. B. hinsichtlich der Fülle und Komplexität an aufgezeichneten Daten sowie deren schwieriger Anonymisierung (Schnettler \& Raab 2008). Forschende fokussieren mit dem zweiten Auswertungsmodus die Analyse des Zeichenprodukts in einer quantitativen oder qualitativen Art und Weise. Ausgewertet werden können beispielsweise die Anzahl und die Art der Orte, Wege und Objekte auf der Karte sowie deren Größe, Position und Entfernung zueinander, basierend auf der Annahme, dass dies für die Teilnehmenden beim Zeichnen eine Rolle spielte (Lutz et al. 2003). Insbesondere hinsichtlich der Eigenschaften von Orten können Beschriftungen auf der Karte, Notizen und vor allem Erklärungen, die in Audioaufnahmen festgehalten sind, benutzt werden (ebd.; Sampson \& Gifford 2010). Daraus lassen sich Bewertungen des $\mathrm{Zu}$ gangs und Nicht-Zugangs zu Orten herausarbeiten. Der dritte Auswertungsmodus kombiniert den prozess- und produktorientierten Modus und ermöglicht damit eine synthetisierende Analyse der gewonnenen Daten (vgl. Lutz et al. 2003).

Herausforderungen in der Forschungspraxis ergaben sich in der Fallstudie durch hohe Personalressourcen (mind. zwei Forschende plus Dolmetscher*in) und einen beachtlichen Zeitbedarf (zwei bis drei Stunden). Teilnehmende sollten zudem bereits einige Zeit am Ort leben, da sie nur dann Alltagserfahrungen gemacht haben und den Zugang zu Orten bewerten können. Schwierigkeiten bei der Durchführung von Mobility Mapping traten beispielsweise auch dann auf, wenn Personen in der Vergangenheit relativ wenige Erfahrungen mit offenen Befragungsformen und freier Meinungsäußerung gemacht hatten. Antworten fielen in diesem Fall sehr knapp aus und erforderten einen engen Frage-Antwort-Zyklus, der wiederum keine kohärenten und langen, zusammenhängenden Erzählungen bzw. Narrative zu Tage förderte. Dadurch besteht die Gefahr, dass das $\mathrm{Mo}$ bility Mapping sehr stark forscher*innengeleitet wird (Kordel et al. 2019). Daneben mag auch eine fehlende Vertrautheit mit visualisierenden Techniken in eingeschränkten Zeichenfähigkeiten der Teilnehmenden oder Kompetenzängsten zu zeichnen oder zu schreiben resultiert haben, die durch eine kontinuierliche Ermutigung seitens der Interviewenden überwunden werden konnte. Schließlich konnte die vertiefte Reflexion der Teilnehmenden über ihre eigene Lebenswelt aber auch fehlende Handlungsfähigkeit (Agency) sichtbar machen, etwa bei der Diskussion über nichtzugängliche Orte.

\section{Mobility Mapping im Geographieunter- richt}

Visuelle Methoden wurden in der Vergangenheit bereits vielfach mit Kindern und Jugendlichen durchgeführt (vgl. Matthews 1984; Morrow 2001; Lutz et al. 2003; Gifford et al. 2007). Bei der Übertragung der Forschungsmethode des Mobility Mapping in den Geographieunterricht eignet sich eine inhaltliche Fokussierung auf, erstens, die Lebenswelten und die relevanten Orte von Jugendlichen. Mit dem Prinzip der Schüler*innenorientierung geht eine Motivation der Schüler*innen einher. Zweitens wird methodisch die Erstellung und Auswertung einfacher, kognitiver Karten fokussiert und in einer weiteren, sich anschließenden, aber hier nicht näher ausgeführten Unterrichtssequenz die Bewertung von Raumkonzepten thematisiert.

Mit der Erstellung von Mobility Maps wird der Kompetenzbereich Erkenntnisgewinnung / Methoden angesprochen (DGfG 2014). In Bezug auf räumliche Orientierung erwerben Schüler*innen zudem Kartenkompetenzen, orientieren sich in ihren Nahräumen und reflektieren über Raumwahrnehmungen. Mobility Maps bieten vor allem jüngeren Schüler*innen die Möglichkeit, sich im Nahraum $\mathrm{zu}$ orientieren und mit den eigenen Bewegungsmustern auseinanderzusetzen. Durch die Reflexion von Schwierigkeiten, bestimmte Orte zu erreichen, entwickeln sie auf spielerischem bzw. kreativem Weg problemlösungsorientierte Kompetenzen, die in Handlungsansätze für bewusstere und nachhaltigere Alltagspraktiken münden können. Außerdem werden Unterstufenschüler*innen durch Mobility Maps an die Daseinsgrundfunktionen „leben“, „wohnen“, „sich versorgen“, „sich bilden“, „sich erholen“ und „am Verkehr teilnehmen“ herangeführt.

Wenn Kartenprodukte und die begleitenden Narrative von Schüler*innen nebeneinandergestellt werden, erkennen die Schüler*innen Unterschiede in Raumwahrnehmungen, zum Beispiel im Hinblick auf die Relevanz von Orten oder Erreichbarkeiten. Schließlich können Erkenntnisse aus dem Mobility Mapping zur Gemachtheit von Räumen in die Diskussion über Raumkonzepte einführen. Somit erwer- 
ben speziell Schüler*innen der Sekundarstufe I gemäß des Lehrplans Geographie durch Mobility Mapping die Kompetenz, die räumliche Strukturiertheit der Umwelt bewusst wahrzunehmen, indem sie diese durch die ihrem Alter entsprechend relativ einfach gehaltene graphische Umsetzung ihres persönlichen Bewegungs- und Raumnutzungsprofils festhalten (BGBl 2021). Schüler*innen der Sekundarstufe II werden durch die Arbeitstechnik des Mobility Mappings vor allem in ihrer Kompetenz gefördert, ihre alltagsweltliche Wahrnehmung des Raumes bewusst zu reflektieren und kritisch zu hinterfragen (BGBl 2021; vgl. „Fähigkeit zur Reflexion von Raumwahrnehmung und -konstruktion“, DGfG 2014).

\section{Durchführung des Mobility Mapping als jahrgangsstufenübergreifende, projektar- tige Lehr-Lern-Sequenz}

Der Einsatz von Mobility Mapping im Unterricht wird im Folgenden als jahrgangsstufenübergreifende, projektartige Lehr-Lern-Unterrichtssequenz entwickelt. Zielgruppe 1 sind Schüler*innen der Oberstufe, Zielgruppe 2 sind Schüler*innen der Unterstufe. Ersteren wird in diesem Projekt die Aufgabe zuteil, dass sie als Lehrende agieren und dabei kognitive, instrumentelle und sozial-affektive Kompetenzen erwerben. Methodisch erlernen sie die Vorbereitung, Durchführung und Auswertung kartenbezogener Erhebungsmethoden. Sie reflektieren dabei über die einzelnen Schritte der Erkenntnisgewinnung und sind in der Lage diese zu beschreiben. Im Rahmen der Präsentation der Ergebnisse vertiefen die Schüler*innen der Zielgruppe 1 ihre Fertigkeiten zum selbstständigen Anfertigen von Strukturskizzen und zum Auswerten von Wirkungsgefügen. Mithilfe des Mobility Mappings werden wichtige Orte, soziale Praktiken und schließlich Raumkonstruktionen aus Sicht der Schüler*innen fokussiert. Insbesondere sollen räumliche Kontexte (städtischländlich), aber auch individuumsbezogene Konstellationen (sozio-demographische, sozio-ökonomische, Zugang zu Mobilität) identifiziert werden. Dabei wird der Frage nachgegangen werden, wie der Lebensraum am eigenen Wohnort von Schüler*innen, gemacht und erfahren wird und welche relationalen Bezüge (räumlich, zeitlich) hergestellt werden. Durch das Auswerten der Mobility Maps der jüngeren Schüler*innen ist die Zielgruppe 1 in der Lage, räumliche Strukturen anhand von individuellen Lebenswelten zu bewerten. Letzteres erfolgt durch einen Vergleich der Kartenprodukte: Schüler*innen erkennen, dass unterschiedliche Orte wichtig sind, dass sich die Erreichbarkeit unterscheidet oder zeitliche Aspekte eine Rolle spielen. Die Reflexion dieser Unterschiede kann schließlich Hin- weise auf unterschiedliche Konstruktionen von (Alltags-) Räumen liefern.

Bei der Zielgruppe 2 wiederum können die Mobility Maps als Methode genutzt werden, um Schüler*innen an geographische Arbeitsweisen, wie zum Beispiel das Erstellen einfacher Skizzen und Karten heranzuführen. Die jüngeren Schüler*innen erstellen unter Anleitung die Mobility Maps, erzählen den Oberstufenschüler*innen von ihren Mobilitätserfahrungen und gehen dabei auf die aufgesuchten Orte, die Gründe des Aufsuchens und den Wegen dahin ein. Bei den Schüler*innen der Unterstufe wird auf diese Weise eine Selbstreflexion im Hinblick auf individuell bedeutsame Orte, den eigenen Bewegungsradius und dessen Veränderung im Jugendalter (Aktivitäten mit und ohne Eltern) angestrebt. Dies wird im Prozess der Erstellung einer Karte mithilfe von leitenden Fragen stimuliert. Die Schüler*innen lernen ihre eigene Raumwahrnehmung mittels Mobility Maps kennen und orientieren sich in ihrem Nahraum. Die räumliche Anordnung von Orten (Position/Distanz) wird dabei eingeübt. Aber auch affektive Kompetenzen können erreicht werden, da durch das Erstellen der Mobility Maps die Wissbegierde und die Freude am Entdecken gefördert wird. So konzentrieren sie sich mithilfe der von ihnen erstellen Skizzen auf die für sie relevanten Orte und nehmen diese auf einer neuen Ebene wahr.

Als jahrgangsübergreifende Unterrichtssequenz hat das Mobility Mapping Projektcharakter. Grundsätzlich orientiert sich die Planung an den sechs von Reinfried (2015) beschriebenen Merkmalen eines Projektunterrichts. Zunächst wird von der Lehrkraft die Projektinitiative skizziert, indem sie das Thema festgelegt, welches hier für beide Zielgruppen unter dem Motto „Mein Leben in der Stadt/Region XYZ - unser Leben in der Stadt/Region XYZ?" steht. Den Schüler*innen wird das Ziel der Unterrichtssequenz vorgestellt, die Lebenswelt einzelner Personen zu erfassen und zu verstehen, mit räumlichen Strukturen und Praktiken in Verbindung zu setzen und diese dann schließlich mit anderen Lebenswelten zu vergleichen. Im nächsten Schritt wird die Projektskizze erstellt, indem das Thema konkretisiert und die organisatorischen Rahmenbedingungen festgelegt werden. Hierzu zählt vor allem, dass von der Lehrperson vorgegeben wird, dass man sich mithilfe von Mobility Mapping mit dem Nahraum beschäftigt. Die organisatorische Struktur in Form der jahrgangsübergreifenden Ausrichtung und die Zuordnung von Zielgruppe 1 in Teams zu 3 Personen wird den Schüler*innen ebenfalls mitgeteilt. Den 3er-Teams werden von der Lehrkraft Gruppen von fünf bis sechs Unterstufenschüler*innen zugewiesen. Im Anschluss folgt der Projektplan. In diesem Abschnitt weist die Lehrkraft zunächst die 
Oberstufenschüler*innen in einem Vortrag in den Ablauf eines Forschungsprozesses mit den Phasen Vorbereitung - Durchführung - Auswertung sowie die Methode Mobility Mapping ein. Danach wird zusammen mit den Oberstufenschüler*innen ein grober Ablaufplan nach den oben genannten Phasen erstellt und es werden Rollen in den 3er-Teams verteilt (siehe Anhang: Arbeitsmaterial 1). Des Weiteren werden eine Protokolltabelle und eine Materialliste erarbeitet, die für jedes Mobility Mapping ein DIN A1 Papier, Filzstifte in vier Farben sowie weiße, gelbe und grüne quadratische Kärtchen und einen Klebestift enthält (siehe Anhang: Arbeitsmaterial 2 und 3). Nun folgt die eigentliche Projektdurchführung. Sie beginnt für die Zielgruppe 1 mit der Vorbereitung des Mobility Mappings. Gruppenintern wird zunächst überlegt, welche Frageimpulse sich für die jüngeren Schüler*innen für das Erstellen einer Mobility Map im Hinblick auf die eigene Lebenswelt und den Nahraum eignen. Dem Prinzip der Schüler*innenorientierung folgend werden die Lebensbereiche Freizeit und Versorgung fokussiert. Im Unterrichtsgespräch einigt man sich gemeinsam auf einen Fragenkatalog. Die Oberstufenschüler*innen sollen dafür sensibilisiert werden, dass es nicht nur um eine quantitative Erfassung von Orten, sondern insbesondere um Bedeutungszuschreibungen geht. Dementsprechend sind sie angehalten, erzählgenerierende Frageimpulse zu geben und nachzufragen, warum Orte aufgesucht werden (vgl. Beispiel in Tab. 1).

Tab. 1: Mögliche erzählgenerierende Frageimpulse der Schüler*innen (eigene Darstellung)

1 Erzählt uns bitte am Anfang darüber, wo ihr Offener Einstieg an einem normalen Schultag hingeht? Was macht ihr dort?

2 Geht ihr an bestimmte Orte, um Sport zu treiDifferenzierung ben? Wo übt ihr andere Freizeitakivitäten aus?

nach Aktivitäten 3 Wo kauft ihr Essen ein? Wo geht ihr essen? Erzählt uns ein bisschen darüber, wie es dort ist!

Differenzierung nach sozialen Kontakten /

Zeiten

Differenzierung nach Distanzen

Differenzierung nach Verkehrsmitteln

Differenzierung nach (Nicht-) Erreichbarkeit
4 Gibt es Orte, die ihr mit den Eltern aufsucht? Welche sind das?

5 Nun dürft ihr einmal an das Wochenende denken. An welche Orte/Plätze geht/fahrt ihr nur am Wochenende?

6 Wie weit sind die Orte von eurem Wohnort oder Schulort entfernt? Ordnet die Kärtchen mit den Orten entsprechend ihrer Distanz auf der Karte an.

7 Wie kommt ihr zu den verschiedenen Orten/Plätzen? Welche Verkehrsmittel nutz† ihr?

8 Wenn ihr eure Karte am Ende nochmal anseht, gibt es auch Orte / Plätze, zu denen ihr nicht kommt, z. B. weil ihr nicht hin gehen dürft, weil es zu teuer ist dort hinzukommen oder aus anderen Gründen?
In einer Doppelstunde leiten die Oberstufenschüler*innen in 3er-Teams die jüngeren Schüler*innen bei der Erstellung der Mobility Maps an und führen insgesamt je fünf Kartierungen durch. Dabei sind die Rollen der Fragensteller*innen, Protokollant*innen und Personen, die Materialien bereithalten, vorgesehen. Das Erstellen der Karten erfolgt in Form einer arbeitsgleichen Gruppenarbeit, in der jede*r ihre*seine eigene Karte erstellt. Als Ausgangspunkte werden sowohl der Wohn- als auch der Schulort vorgegeben. Zunächst werden Orte zu den Frageimpulsen 1-3 im Gespräch identifiziert und auf weißen Kärtchen notiert. Es folgen Orte, die nur mit den Eltern aufgesucht werden (Frageimpuls 4, gelbe Kärtchen). Auf den grünen Kärtchen werden Orte festgehalten, die von den Schüler*innen am Wochenende besucht werden (Frageimpuls 5). Wichtig ist, dass darauf geachtet wird, dass alle Aktivitäten immer mit Ortsangaben versehen werden. Anschließend wird in den Kleingruppen die wahrgenommene Distanz zwischen den Orten und dem Wohnort oder Schulort erarbeitet (vgl. Frageimpuls 6). Hierfür ist vorteilhaft, dass die Kärtchen bei Bedarf flexibel verschiebbar sind. Dann können diese fixiert werden. Um in der Skizze deutlich zu machen, wie die Schüler*innen an die jeweiligen Orte/Plätze gelangen, also ob zu Fuß, mit dem Fahrrad, dem Auto oder mit öffentlichen Verkehrsmitteln zeichnen sie mithilfe der vier Filzstifte Verbindungslinien zwischen Start- und Zielorten (vgl. Frageimpuls 7). Abschließend werden nichterreichbare Orte abgefragt (vgl. Frageimpuls 8) und sozialstatistische Daten wie zum Beispiel Wohnort, Verfügbarkeit eines Fahrrads, Autos im Haushalt etc. erfasst. Der Lehrkraft kommt in dieser Phase eine beratende Rolle zu. Im Rahmen von Zwischengesprächen klärt sie sachlich-inhaltliche Fragen und unterstützt bei Problemen.

Im weiteren Verlauf wertet die Zielgruppe $1 \mathrm{zu} \mathrm{Hau}-$ se die Mobility Maps der Unterstufenschüler*innen in den jeweiligen 3er-Teams aus. Diese Auswertung erfolgt in einer quantitativen und qualitativen Art und Weise, indem die Karten anhand verschiedener Kategorien betrachtet werden, z. B. Art der aufgesuchten Orte/ Plätze und Bedeutungen für die Schüler*innen (wohin und warum?), mit und ohne Begleitung der Eltern aufgesuchte Orte / Plätze (mit wem?), zeitliche Frequentierung der aufgesuchten Orte / Plätze (werktags, Wochenende) (wann?), genutzte Fortbewegungsmittel (wie?) und Aktionsradius (siehe Arbeitsmaterial 3). Das Protokoll mit den Erklärungen zu Orten wird dafür herangezogen. Im Anschluss werden die Ergebnisse der einzelnen Schüler*innen, die von den 3er-Teams betreut wurden, verglichen, um Einblicke in die Unterschiedlichkeit von Lebenswel- 
ten und sozialen/räumlichen Praktiken zu erhalten. Mögliche Aspekte des Vergleichs sind:

- aufgesuchteOrte nach Wohnort der Schüler*innen (z. B. Stadt - Land)

- aufgesuchte Orte nach Verfügbarkeit von Verkehrsmitteln der Schüler*innen

- Bedeutungen von Orten nach individuellen Merkmalen (Zeit, Interessen, Erlaubnis von Eltern)

- Bedeutung von Wegen (teuer, nicht erlaubt, lange Fahrtzeiten, ...)

Die Ergebnisse werden von den einzelnen 3er-Teams präsentiert und im Unterrichtsgespräch diskutiert. Im Rahmen der Präsentationen sind die Schüler*innen angehalten, ihr Vorgehen transparent zu machen, d. h. die Stichprobe ihrer Befragung darzustellen und über ihre Erfahrungen bei der Durchführung zu berichten. Dabei sollen explizit soziale und affektive Lernzieldimensionen stimuliert werden.

Als Projektabschluss stellen die 3er-Teams ihren Unterstufengruppen die Ergebnisse mittels der Tabellen vor und geben die Karten an die Ersteller*innen zurück. Die Lehrkraft führt abschließend ein Unterrichtsgespräch mit der Unterstufenklasse, um diesen die Möglichkeit zu geben ihre eigene Raumwahrnehmung und -nutzung zu reflektieren.

\section{Chancen der Methode aus fachwissen- schaftlicher und fachdidaktischer Perspektive}

Das als partizipative Forschungsmethode konzipierte Mobility Mapping greift ein verändertes Rollenverständnis von Forschenden und Teilnehmenden auf. Ersteren kommt dabei eher eine unterstützende Funktion als ,Katalysator*innen' zu, Letztere hingegen nehmen aktiv am Forschungsprozess teil und werden dabei dazu befähigt, Bedeutungen von Orten, soziale Praktiken und die Bewertung dieser in Kategorien und Begriffen aus ihrer Lebenswelt zu erklären. Mobility Mapping bietet zudem eine Artikulationsmöglichkeit, die weniger stark von Sprache und Lese- bzw. Schreibfähigkeiten abhängt (Täubig 2009). Gegenüber anderen multi-sensuellen Interviewformen wie Fotointerviews oder walking interviews (siehe Beitrag von Langner, Naumann \& Raschke im vorliegenden Heft), bei denen Teilnehmende Forschenden individuell bedeutsame Orte zeigen oder gemeinsam mit ihnen Wege ablaufen, bietet Mobility Mapping zudem weitere Vorteile. So sind bei Mobility Mapping keine technischen Trainings notwendig, Herausforderungen des Datenschutzes oder Urheberrechts wie etwa beim Fotogra- fieren von Personen sind weniger stark ausgeprägt und das Mobility Mapping ist weniger zeitaufwändig und ortsunabhängiger durchführbar.

Mit der Übertragung der Methode des Mobility Mapping von der Fachwissenschaft in die schulische Unterrichtspraxis lassen sich auch zahlreiche Chancen und Vorteile transferieren. So kommt den Teilnehmenden beim Mobility Mapping sowohl eine große Selbstständigkeit und Entscheidungsmacht über die Inhalte und deren Wertung als auch ein eigener Handlungsspielraum in Bezug auf die Durchführung und die visuelle Darstellung zu. Damit kann in der Unterrichtspraxis auf die präferierten Darstellungsmöglichkeiten von Schüler*innen eingegangen werden. Sie erhalten außerdem Kompetenzen von „Forschenden“ und haben selbst Teil an dieser Rolle. Insbesondere für Schüler*innen stellt die Erstellung bzw. die Anleitung zu dieser und Auswertung von Karten, hier subjektiver Karten (mental maps), einen wichtigen Moment der Reflexion über die Lebenswelten von anderen Menschen und den räumlichen Strukturen dar. Individuelle und strukturelle Voraussetzungen für das Erreichen von Orten können ebenfalls reflektiert werden, da das Instrument des (Nicht-)Zugangs zu Orten erfasst und Voraussetzungen für den Zugang identifiziert (z. B. die Begleitung durch Eltern). Außerdem kann gemäß der Lehrpläne der Sekundarstufe I und II das Erstellen der Mobility Maps mit der Kernkompetenz des Kartenlesens im Geographieunterricht verknüpft werden. Lehrer*innen können die Ergebnisse des Mobility Mappings nutzen, um mit den Oberstufenschüler*innen im Anschluss über neuere Raumkonzepte, wie die Gemachtheit von Räumen zu diskutieren und über ein relatives Verständnis von Räumen zu reflektieren. Indem unterschiedliche Bedeutungen von Orten nebeneinandergestellt werden, können Annahmen über die Relevanz und Rollen dieser Orte im Lebensalltag von Personen kritisch reflektiert und ggf. validiert oder modifiziert werden.

\section{Danksagung}

Die Autor*innen danken dem Open Access Publikationsfonds der FAU - Friedrich-Alexander Universität Erlangen-Nürnberg - für die Unterstützung.

Darüber hinaus geht ein herzlicher Dank an die Gastherausgeber*innen für die Einladung zum Themenheft und die Koordination des Prozesses sowie zwei anonyme Gutachter*innen für die wertvollen Kommentare zu früheren Versionen des Textes. Sie halfen dazu, den Artikel substanziell zu verbessern. 


\section{Literaturverzeichnis}

BGBl. (Bundesgesetzblatt für die Republik Österreich): Bundesrecht konsolidiert: Gesamte Rechtsvorschrift für Lehrpläne - allgemeinbildende höhere Schulen, Fassung vom 10.08.2021. Rechtsinformationssystem des Bundes (RIS), Wien. https://www.ris.bka.gv.at/GeltendeFassung.wxe?Abfrage=Bundesnormen $\&$ Gesetzesnumm er $=10008568$ (10.08.2021).

Collier, J. \& M. Collier (1986): Visual Anthropology: Photography as a Research Method. University of Mexico Press, Albuquerque.

DGfG (Deutsche Gesellschaft für Geographie) (2014): Bildungsstandards im Fach Geographie für den Mittleren Schulabschluss mit Aufgabenbeispielen. Selbstverlag, Bonn.

Gifford, S. M., C. Bakopanos, I. Kaplan \& I. Correa-Velez (2007): Meaning or Measurement? Researching the Social Contexts of Health and Settlement Among Newlyarrived Refugee Youth in Melbourne, Australia. In: Journal of Refugee Studies 20(3). S. 414-440.

Honer, A. (2011): Lebenswelt. In: Bohnsack, R., Marotzki, W. \& M. Meuser (Hrsg.): Hauptbegriffe qualitativer Sozialforschung. Barbara Budrich, Opladen/Farmington Hills. S. 110-112.

Kordel, S. (2015): Striving for the „good life“ - Homemaking among senior citizens on the move. An analysis of German (pre)retirees in Spain and Germany in a continuum of tourism and migration. Dissertation, Universität Erlangen-Nürnberg.

Kordel, S., T. Weidinger \& S. Hachmeister (2019): Benachteiligungen begegnen: Mobility Maps als visuell-partizipatives Tool zur Analyse von Lebenswelten geflüchteter Menschen in ländlichen Räumen. In: Mießner, M. \& M. Naumann (Hrsg.): Kritische Geographien ländlicher Entwicklung. Globale Transformationen und lokale Herausforderungen. Westfälisches Dampfboot, Münster. S. 279-292.

Kumar, S. (2002): Methods for Community Participation. A Complete Guide for Practitioners. ITDG Publishing, London.

Lutz, M., I. Behnken \& J. Zinnecker (2003): Narrative Landkarten. Ein Verfahren zur Rekonstruktion aktueller und biographisch erinnerter Lebensräume. In: Friebertshäuser, B. \& A. Prengel (Hrsg.): Handbuch Qualitative Forschungsmethoden in der Erziehungswissenschaft. Juventa, Weinheim/München. S. 414-435.
Matthews, M. H. (1984): Environmental Cognition of Young Children: Images of Journey to School and Home Area. In: Transactions of the Institute of British Geographers 9(1). S. 89-105.

Morojele, P. \& N. Muthukrishna (2012): The Journey to School: Space, Geography and Experiences of Rural Children. In: Perspectives in Education 30(1). S. 90-100.

Morrow, V. (2001): Using Qualitative Methods to Elicit Young People's Perspectives on Their Environment. In: Health Education Research 16(3). S. 255-268. DOI: 10.1093/her/16.3.255

Reinfried, S. (2015): Projekt. In: Reinfried, S. \& H. Haubrich (Hrsg.): Geographie unterrichten lernen. Die Didaktik der Geographie. Cornelsen, Berlin. S. 176-177.

Rinschede, G. \& A. Siegmund (2020): Geographiedidaktik. utb, Stuttgart.

Rose, G. (2007): Visual Methodologies: An Introduction to the interpretation of Visual Methods. SAGE, London.

Sampson, R. \& S. M. Gifford (2010): Place-making, Settlement and Well-being: The Therapeutic Landscapes of Recently Arrived Youth with Refugee Backgrounds. In: Health \& Place 16(1). S. 116-131. DOI: 10.1016/j. healthplace.2009.09.004

Schlottmann, A. \& J. Miggelbrink (2009): Visuelle Geographien - ein Editorial. In: Social Geography 4(1). S. 13-24. DOI: $10.5194 / \mathrm{sg}-4-13-2009$

Schnettler, B. \& J. Raab (2008): Interpretative Visual Analysis. Developments, State of the Art and Pending Problems. In: Forum: Qualitative Sozialforschung/Forum: Qualitative Social Research 9(3), Art. 31. DOI: 10.17169/fqs-9.3.1149

Schütz, A. \& T. Luckmann (1979). Strukturen der Lebenswelt. Suhrkamp, Frankfurt/M.

Spencer, S. (2011): Visual Research Methods in the Social Sciences: Awakening Visions. Routledge, London.

Täubig, V. (2009): Totale Institution Asyl. Empirische Befunde zu alltäglichen Lebensführungen in der organisierten Desintegration. Juventa, Weinheim/München.

Weidinger, T., S. Kordel \& J. Kieslinger (2019/2021): Unravelling the meaning of place and spatial mobility: Analysing the everyday life worlds of refugees in host societies by means of mobility mapping. In: Journal of Refugee Studies 34(1). S. 374-396. DOI: 10.1093/jrs/fez004

Wilde, M. (2014): Mobilität und Alltag. Einblicke in die Mobilitätspraxis älterer Menschen auf dem Land. Springer VS, Wiesbaden. 


\title{
Material 1: Rollenverteilung
}

\begin{abstract}
Fragensteller*in: Diese*r Schüler*in nimmt die Rolle der Person ein, die hauptsächlich die Fragen stellt und sollte deshalb mit den Hauptthemen des Leifadens gut vertraut sein. Sie*er sollte zudem darauf achten, erzählgenerierende Fragen zu stellen (ja/nein Fragen vermeiden), die Unterstufenschüler*innen ausreden zu lassen aber gleichzeitig stets interessiert nachzufragen (Warum gehst du dorthin?, Was gefällt dir besonders dort?).
\end{abstract}

Protokollant*in: Sie*er ist dafür verantwortlich, die Aussagen möglichst detailgetreu im Protokoll zu erfassen, ohne diese zu stark zu interpretieren. Ist etwas unklar, kann die*der Protokollant*in unterbrechen und Nachfragen anstellen, hält sich aber während des Gesprächs selbst eher zurück. Nach der Durchführung des Mobility Mappings spricht sie*er mit den anderen beiden Schüler*innen und klärt offene Fragen.

\footnotetext{
Materialverantwortliche*r: Der*die Schüler*in trägt dafür Verantwortung, dass zu Beginn des Gesprächs alle Materialien der Liste in ausreichender Anzahl zur Verfügung stehen. Sie*er reicht den Unterstufenschüler*innen zur richtigen Zeit die richtigen Materialien und entlastet damit die*denFragensteller*in.
}

\section{Material 2: Materialliste Interviews}

Für jede*n Schüler*in wird benötigt:

- Leitfaden und Protokollvorlage für alle Interviewenden

- 2 weiße DIN A1 Plakate (Standard Flipchart-Blätter, 1 Reserve)

- Weiße eckige Karten für Orte, die alleine besucht werden $(10 \times 10 \mathrm{~cm})$

- Gelbe eckige Karten für Orte, die mit den Eltern besucht werden $(10$ x $10 \mathrm{~cm})$

- Grüne eckige Karten für Orte, die am Wochenende besucht werden $(10 \times 10 \mathrm{~cm})$

- 4 verschiedenfarbige, dicke Filzstifte für unterschiedliche Verkehrsmittel

- Klebestift, Klebeband 


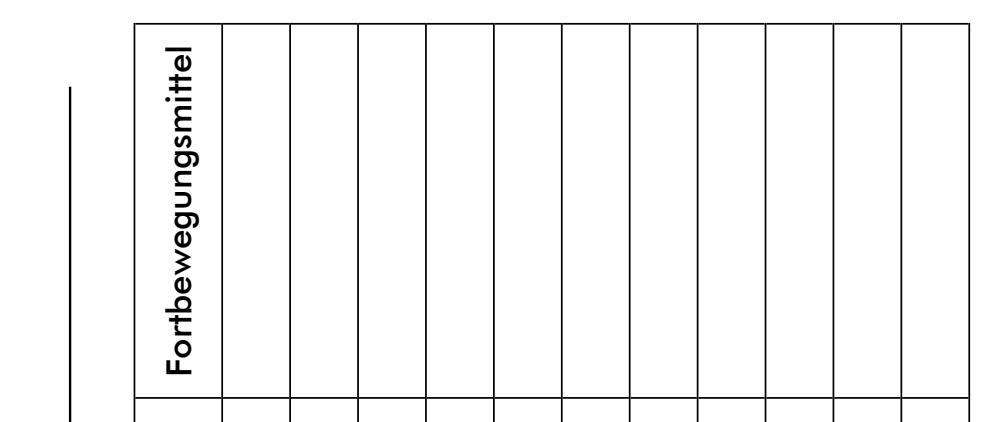

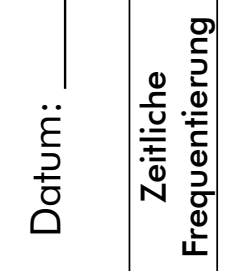

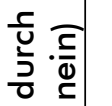

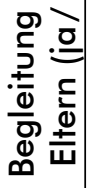

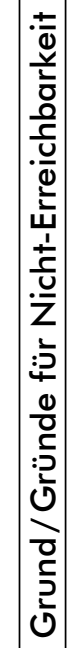

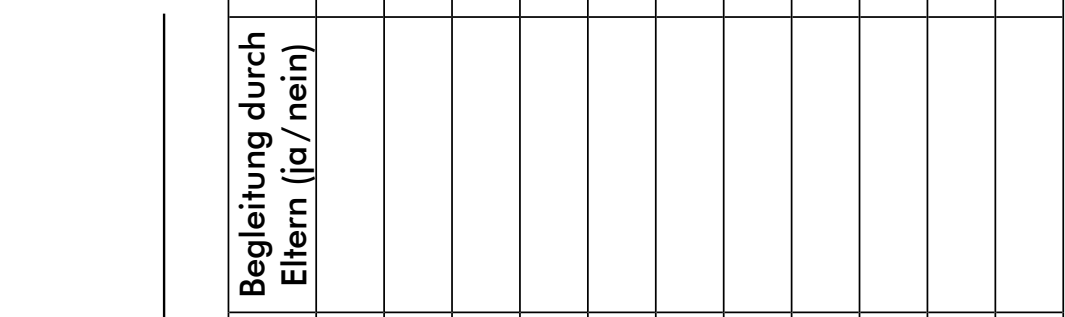

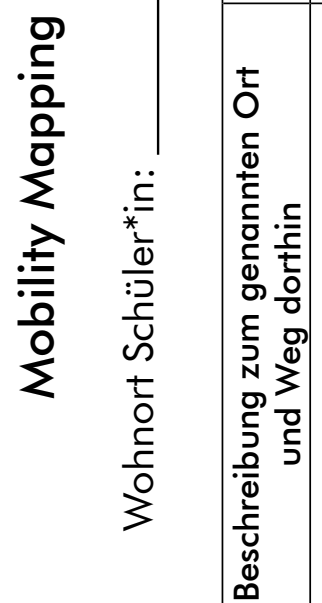

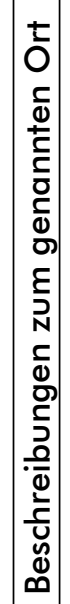
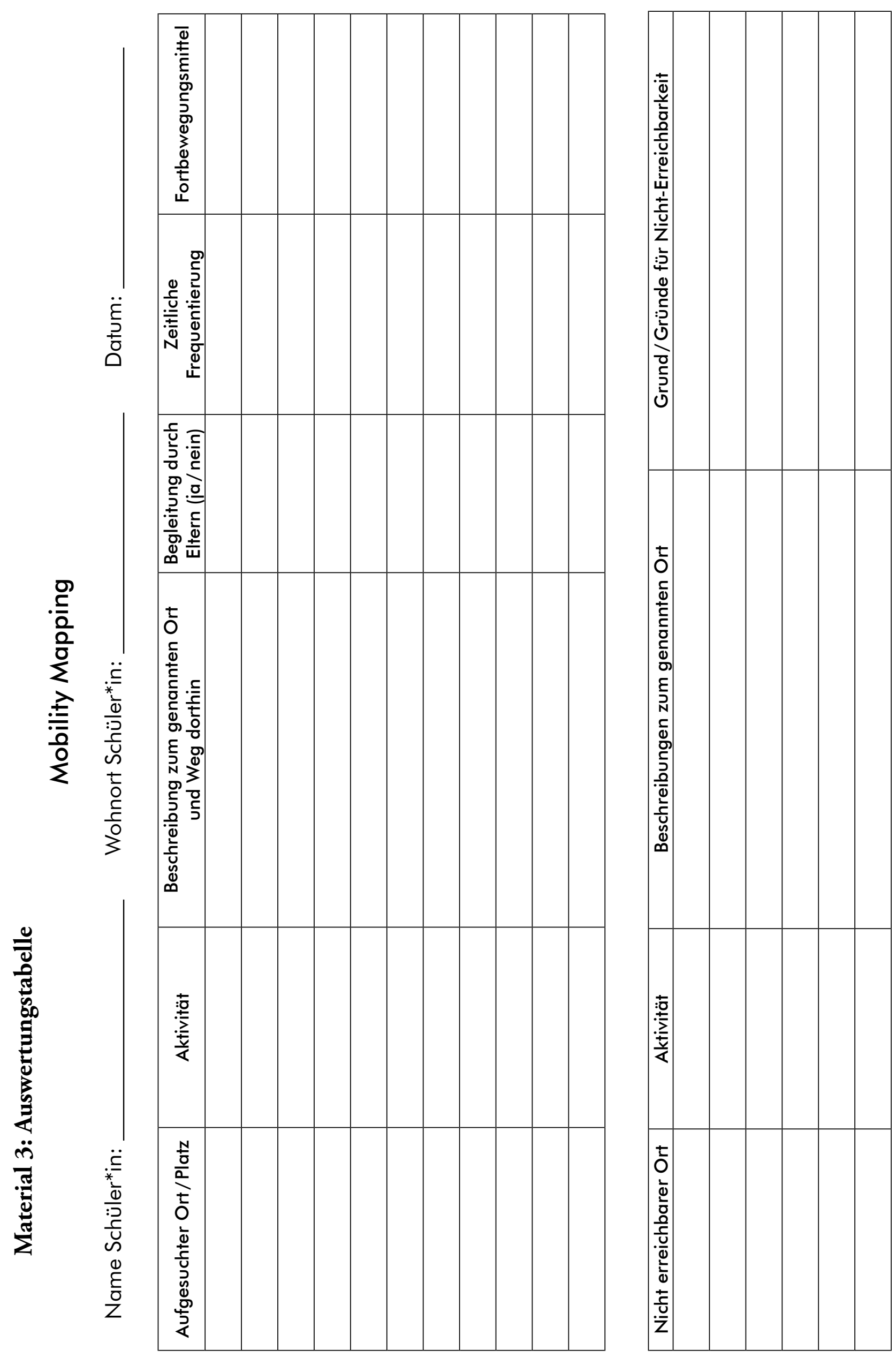

苦

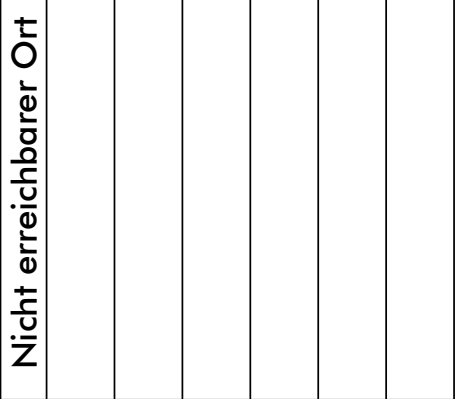

